\title{
WIDELY LINEAR EQUALIZATION OF ROTATIONALLY VARIANT SIGNALS
}

\author{
Pei Xiao, Colin Cowan \\ Institute of ECIT, Queen's University Belfast \\ Queen's Road, Belfast, BT 3 9DT, UK \\ email: \{pei.xiao,c.cowan\}@ecit.qub.ac.uk
}

\author{
Honggang Zhang \\ Department of ISEE, Zhejiang University \\ Zheda Road 38, Hangzhou 310027 \\ email: honggangzhang@zju.edu.cn
}

\begin{abstract}
Several widely linear equalization algorithms utilizing the rotationally variant nature of the received signals are presented in this paper to combat the detrimental effect of intersymbol interference (ISI) introduced by frequency selective channels. Their adaptive implementations and application to the time-reversal space-time coded (TR-STBC) system are also considered. In addition, a widely linear approach to turbo equalization is derived for systems employing error correction code. The widely linear equalizers and turbo equalizer are evaluated over broadband fixed wireless access channels, and are shown to yield superior performance compared to the conventional linear schemes.
\end{abstract}

\section{KEY WORDS}

Equalization, frequency selective channels.

\section{Introduction}

One of the limiting factors in outdoor wireless transmission is the multipath channel between the transmitter and the receiver giving rise to intersymbol interference (ISI), which degrades the system performance and limits the maximum achievable data rate. The problem can be tackled by employing OFDM technology [1], which transforms the frequency selective channel into a number of parallel flat fading channels. Another effective remedy to combat the detrimental effects caused by ISI is the use of equalization, which is the focus of this study.

For a complex random vector $\mathbf{r}$, its second-order averages are completely characterized by its autocorrelation matrix $\mathbf{C}=\mathrm{E}\left[\mathbf{r r}^{H}\right]$ as well as the pseudo-autocorrelation matrix $\tilde{\mathbf{C}}=\mathrm{E}\left[\mathbf{r r}^{T}\right]$ [2]. The superscript operators ()$^{H},()^{*},()^{T}$ denote the conjugate transpose, conjugate, and transpose operations, respectively. Most existing studies on receiver algorithms only exploit the information contained in the autocorrelation function of the observed signal. The pseudo-autocorrelation matrix $\tilde{\mathbf{C}}$ is usually not considered and is implicitly assumed to be zero. While this is the optimum strategy when dealing with rotationally invariant complex random processes (i.e., when pseudo-autocorrelation $\tilde{\mathbf{C}}$ is vanishing) [3], it turns out to be sub-optimum in situations where the transmitted signals and/or interference are rotationally variant complex random processes (i.e., $\tilde{\mathbf{C}}$ is non-vanishing), for which the performance of a linear receiver can generally be improved by the use of widely linear processing (WLP) [4]. It was shown in [5] that for systems employing a real valued constellation with complex valued channel coefficients, full exploitation of the available information on the second-order statistics of the observations entails the use of WLP, i.e., the received signal and its complex conjugate are separately filtered and results are linearly combined. The results show that the proposed systems outperform the other systems currently known in the literature. The strategy proposed in [5] was employed in [6] for the derivation of widely linear (WL) equalization schemes.

In this paper, we provide a thorough treatment for the widely linear processing of rotationally variant signals. First, we discuss the WL equalization design in an uncoded system, then extend the schemes to a space-time coded system, and finally derive a WL based turbo equalizer which integrates the WL equalizer and a soft-input, soft-output channel decoder in an iterative manner.

\section{WLP for uncoded system}

First, we study the application of WLP in an uncoded system. The baseband equivalent of the transmission system under study is described as follows. The information bits are first mapped into BPSK symbols $\left\{b_{n}\right\}$, which are subsequently transmitted over a frequency selective channel. The channel can be modelled by an equivalent baseband system where the concatenation of the the transmit filter, the channel and the receive filter, is represented by a discrete-time $T$-tap transversal filter with finite-length impulse response $h_{n}=\sum_{t=0}^{T-1} h_{t} \delta_{n-l}$ where $h_{t}$ denotes the complex channel coefficients. The received signal is formed as

$$
r_{n}=\sum_{t=0}^{T-1} h_{t} b_{n-t}+v_{n},
$$

The transmitted symbol at time instant $n$ is denoted as $b_{n}$, and $v_{n}$ is the complex additive white Gaussian noise with zero mean and variance $N_{0}$.

The task of the receiver is to detect the transmitted symbols $\left\{b_{n}\right\}$ given the received observation $\left\{r_{n}\right\}$. From (1), we see that the desired symbol is corrupted with ISI and AWGN. An equalizer is needed to combat ISI and 
to improve the error rate performance. The conventional linear MMSE equalization scheme and its improved versions using WLP will be discussed next.

\subsection{Conventional LMMSE equalization}

The conventional linear MMSE equalizer (with $2 L+1$ taps and detection delay $d$ ) is illustrated in Fig. 1 and is designed to minimize the mean square error (MSE) between the equalizer output $z_{n}$ and symbol $b_{n-d}$ [7]

$$
\epsilon_{n}=\mathrm{E}\left\{\left|z_{n}-b_{n-d}\right|^{2}\right\}=\mathrm{E}\left\{\left|\mathbf{a}^{H} \mathbf{r}_{n}-b_{n-d}\right|^{2}\right\} .
$$

The output $z_{n}$ is formed as $z_{n}=\sum_{k=0}^{2 L} a_{k}^{*} r_{n-k}=$ $\mathbf{a}^{H} \mathbf{r}_{n}$, where $\mathbf{r}_{n}=\left[\begin{array}{llll}r_{n} & r_{n-1} & \cdots & r_{n-2 L+1} \\ r_{n-2 L}\end{array}\right]^{T}$, and $\mathbf{a}=\left[\begin{array}{lllll}a_{0} & a_{1} & \cdots & a_{2 L-1} & a_{2 L}\end{array}\right]^{T}$. The decision is made according to

$$
\hat{b}_{n-d}=\operatorname{sgn}\left(\operatorname{Re}\left\{z_{n}\right\}\right)=\operatorname{sgn}\left(\operatorname{Re}\left\{\mathbf{a}^{H} \mathbf{r}_{n}\right\}\right) .
$$

The coefficients vector $\mathbf{a}$ is computed as [7] $\mathbf{a}=$ $\left(\mathrm{E}\left[\mathbf{r}_{n} \mathbf{r}_{n}^{H}\right]\right)^{-1} \mathrm{E}\left[\mathbf{r}_{n}^{H} b_{n-d}\right]=\mathbf{C}_{\mathbf{r r}}^{-1} \mathbf{C}_{\mathbf{r} b}$, where $\mathbf{C}_{\mathbf{r} b}$ is the cross-correlation vector, and $\mathbf{C}_{\mathbf{r r}}^{-1}$ is the inverse of the autocorrelation matrix $\mathbf{C}_{\mathbf{r r}}$. They are computed as

$$
\mathbf{C}_{\mathbf{r r}}=\mathrm{E}\left[\mathbf{r}_{n} \mathbf{r}_{n}^{H}\right] ; \quad \mathbf{C}_{\mathbf{r} b}=\mathrm{E}\left[\mathbf{r}_{n} b_{n-d}\right]
$$

\subsection{MMSE equalization with WLP}

For the widely linear (WL) MMSE equalizer, the cost function is re-defined as

$$
\epsilon_{n}^{\prime}=\mathrm{E}\left\{\left|z_{n}^{\prime}-b_{n-d}\right|^{2}\right\}=\mathrm{E}\left\{\left|\operatorname{Re}\left\{\mathbf{a}^{H} \mathbf{r}_{n}\right\}-b_{n-d}\right|^{2}\right\}
$$

The reasoning for this modification is that a conventional MMSE filter yields a complex valued filter output. However, only the real part of this output is relevant for the decision in a system with real valued constellation. Minimization of (4) will result in a better estimator than the one designed under the criterion expressed by (2) since $\epsilon_{n}^{\prime}<\epsilon_{n}$ [6]. With this revised MMSE scheme, the decision is made according to

$$
\hat{b}_{n-d}=\operatorname{sgn}\left(z_{n}^{\prime}\right)=\operatorname{sgn}\left(\mathbf{a}^{H} \mathbf{r}_{n}+\mathbf{a}^{T} \mathbf{r}_{n}^{*}\right) .
$$

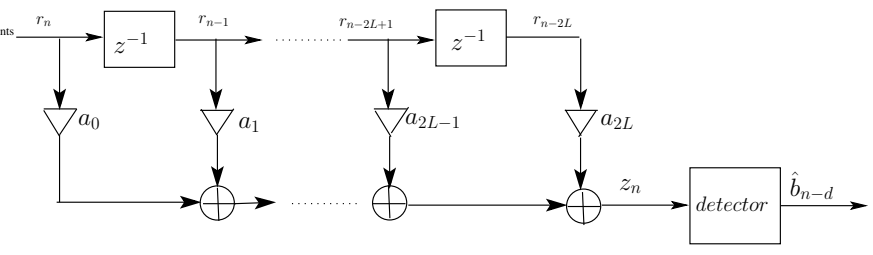

Figure 1. MMSE equalizer with $2 L+1$ taps and detection delay $d$.
We know that

$\operatorname{Re}\left\{\mathbf{a}^{H} \mathbf{r}_{n}\right\}=\frac{1}{2}\left[\mathbf{a}^{H} \mathbf{r}_{n}+\left(\mathbf{a}^{H} \mathbf{r}_{n}\right)^{*}\right]=\frac{1}{2}\left[\mathbf{a}^{H} \mathbf{r}_{n}+\mathbf{a}^{T} \mathbf{r}_{n}^{*}\right]$.

Substituting (5) into (4) yields

$$
\begin{aligned}
\epsilon_{n}= & \mathrm{E}\left\{\left|\operatorname{Re}\left\{\mathbf{a}^{H} \mathbf{r}_{n}\right\}-b_{n-d}\right|^{2}\right\}=\mathrm{E}\left\{\left|\frac{1}{2} \mathbf{a}^{H} \mathbf{r}+\frac{1}{2} \mathbf{a}^{T} \mathbf{r}^{*}-b_{n-d}\right|^{2}\right\} \\
= & \mathrm{E}\left\{\left(\frac{1}{2} \mathbf{a}^{H} \mathbf{r}+\frac{1}{2} \mathbf{a}^{T} \mathbf{r}^{*}-b_{n-d}\right)\left(\frac{1}{2} \mathbf{a}^{H} \mathbf{r}+\frac{1}{2} \mathbf{a}^{T} \mathbf{r}^{*}-b_{n-d}\right)^{*}\right\} \\
= & \frac{1}{4} \mathbf{a}^{H} \mathbf{C}_{\mathbf{r r}} \mathbf{a}+\frac{1}{4} \mathbf{a}^{H} \tilde{\mathbf{C}}_{\mathbf{r r}} \mathbf{a}^{*}-\frac{1}{2} \mathbf{a}^{H} \mathbf{C}_{\mathbf{r} b}+\frac{1}{4} \mathbf{a}^{T} \tilde{\mathbf{C}}_{\mathbf{r r}}^{*} \mathbf{a} \\
& +\frac{1}{4} \mathbf{a}^{T} \mathbf{C}_{\mathbf{r r}}^{*} \mathbf{a}^{*}-\frac{1}{2} \mathbf{a}^{T} \mathbf{C}_{\mathbf{r} b}^{*}-\frac{1}{2} \mathbf{C}_{b \mathbf{r}} \mathbf{a}-\frac{1}{2} \tilde{\mathbf{C}}_{b \mathbf{r}} \mathbf{a}^{*}+\sigma_{b}^{2},
\end{aligned}
$$

where $\mathbf{C}_{\mathbf{r r}}$ and $\mathbf{C}_{\mathbf{r} b}$ are defined in (3) and $\mathbf{C}_{b \mathbf{r}}=$ $\mathrm{E}\left\{b_{n-d} \mathbf{r}_{n}^{H}\right\}, \tilde{\mathbf{C}}_{\mathbf{r r}}=\mathrm{E}\left\{\mathbf{r}_{n} \mathbf{r}_{n}^{T}\right\}$. In the case of transmitting real-valued data over complex-valued channel, the pseudoautocorrelation matrix $\tilde{\mathbf{C}}_{\mathbf{r r}}$ is non-vanishing [6] and utilization of the rotationally variant property of the received signal $\mathbf{r}_{n}$ will consequently lead to a better performance. Differentiating $\epsilon_{n}$ with respect to a and setting it to zero, yields

$$
\mathbf{a}=\left(\mathbf{C}_{\mathbf{r r}}-\tilde{\mathbf{C}}_{\mathbf{r r}} \mathbf{C}_{\mathbf{r r}}^{T-1} \tilde{\mathbf{C}}_{\mathbf{r r}}^{H}\right)^{-1}\left(\mathbf{C}_{\mathbf{r} b}-\tilde{\mathbf{C}}_{\mathbf{r r}} \mathbf{C}_{\mathbf{r r}}^{T-1} \mathbf{C}_{\mathbf{r} b}^{*}\right) .
$$

\subsection{Decision feedback equalizer with WLP}

Here, we discuss how WLP can be applied to decision feedback equalizer (DFE). The output of the WL DFE and cost function are formed as

$$
\begin{aligned}
& z_{n}^{\prime}=\operatorname{Re}\left\{\mathbf{a}^{H} \mathbf{r}_{n}\right\}+\mathbf{g}^{T} \hat{\mathbf{b}}_{n}=\left[\begin{array}{lll}
0.5 \mathbf{a}^{H} & 0.5 \mathbf{a}^{T} & \mathbf{g}^{T}
\end{array}\right]\left[\begin{array}{l}
\mathbf{r}_{n} \\
\mathbf{r}_{n}^{*} \\
\hat{\mathbf{b}}_{n}
\end{array}\right] \\
& =\left[\begin{array}{c}
0.5 \mathbf{a} \\
0.5 \mathbf{a}^{*} \\
\mathbf{g}
\end{array}\right]^{H}\left[\begin{array}{c}
\mathbf{r}_{n} \\
\mathbf{r}_{n}^{*} \\
\hat{\mathbf{b}}_{n}
\end{array}\right]=\boldsymbol{\alpha}_{g}^{H} \mathbf{y}_{n} \\
& \epsilon_{n}=\mathrm{E}\left\{\left|b_{n-d}-z_{n}^{\prime}\right|^{2}\right\}=\boldsymbol{\alpha}_{g}^{H} \mathbf{C}_{\mathbf{y y}} \boldsymbol{\alpha}_{g}-\boldsymbol{\alpha}_{g}^{H} \mathbf{C}_{\mathbf{y} b}-\mathbf{C}_{b \mathbf{y}} \boldsymbol{\alpha}_{g}+\sigma_{b}^{2} \text {, }
\end{aligned}
$$

where $\mathbf{g}=\left[\begin{array}{llll}g_{0} & g_{1} & \cdots & g_{M-1}\end{array}\right]^{T}$ and $\hat{\mathbf{b}}_{n}=$ $\left[\begin{array}{llll}\hat{b}_{n-1} & \hat{b}_{n-2} & \cdots & \hat{b}_{n-M}\end{array}\right]^{T}$ are the feedback filter coefficient vector and the decision feedback vector, respectively. The other vectors and matrices are defined as

$$
\begin{aligned}
& \boldsymbol{\alpha}_{g}=\left[\begin{array}{lll}
0.5 \mathbf{a} & 0.5 \mathbf{a}^{*} & \mathbf{g}
\end{array}\right]^{T} ; \quad \mathbf{y}_{n}=\left[\begin{array}{lll}
\mathbf{r}_{n} & \mathbf{r}_{n}^{*} & \hat{\mathbf{b}}_{n}
\end{array}\right]^{T} \\
& \mathbf{C}_{\mathbf{y y}}=\mathrm{E}\left\{\mathbf{y}_{n} \mathbf{y}_{n}^{H}\right\}=\mathrm{E}\left\{\left[\begin{array}{l}
\mathbf{r}_{n} \\
\mathbf{r}_{n}^{*} \\
\hat{\mathbf{b}}_{n}
\end{array}\right]\left[\begin{array}{lll}
\mathbf{r}_{n}^{H} & \mathbf{r}_{n}^{T} & \hat{\mathbf{b}}_{n}^{H}
\end{array}\right]\right\} \\
& \mathbf{C}_{b \mathbf{y}}=\mathrm{E}\left\{b_{n-d} \mathbf{y}_{n}^{H}\right\}=\mathrm{E}\left\{b_{n-d}\left[\begin{array}{lll}
\mathbf{r}_{n}^{H} & \mathbf{r}_{n}^{T} & \hat{\mathbf{b}}_{n}^{H}
\end{array}\right]\right\} \\
& \mathbf{C}_{\mathbf{y} b}=\mathrm{E}\left\{b_{n-d} \mathbf{y}_{n}\right\}=\mathrm{E}\left\{b_{n-d}\left[\begin{array}{c}
\mathbf{r}_{n} \\
\mathbf{r}_{n}^{*} \\
\hat{\mathbf{b}}_{n}
\end{array}\right]\right\} \text {. }
\end{aligned}
$$

Setting $\frac{\partial \epsilon_{n}}{\partial \boldsymbol{\alpha}_{g}}=\left(\mathbf{C}_{\mathbf{y y}} \boldsymbol{\alpha}_{g}\right)^{*}-\mathbf{C}_{b \mathbf{y}}^{T}$ to zero yields the optimum filter coefficients vector $\boldsymbol{\alpha}_{g}=\mathbf{C}_{\mathbf{y y}}^{-1} \mathbf{C}_{\mathbf{y} b}$. 


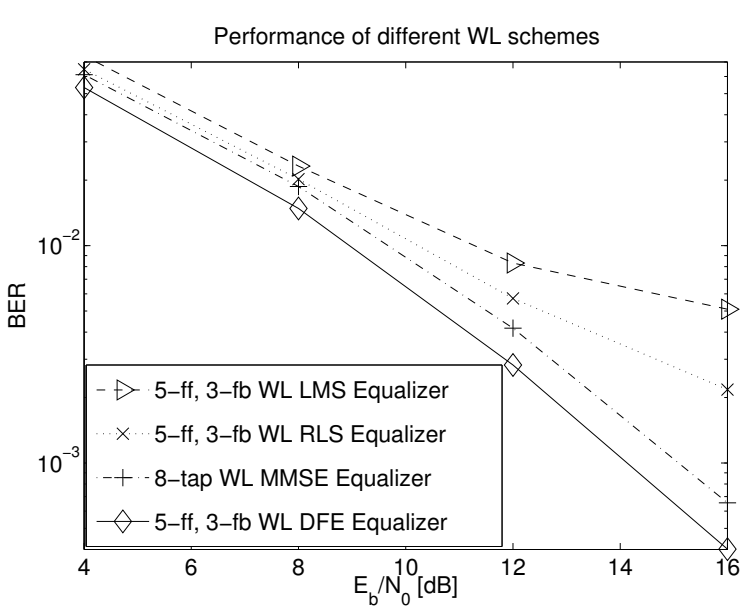

Figure 4. Performance of different WL equalizers for BFWA channel.

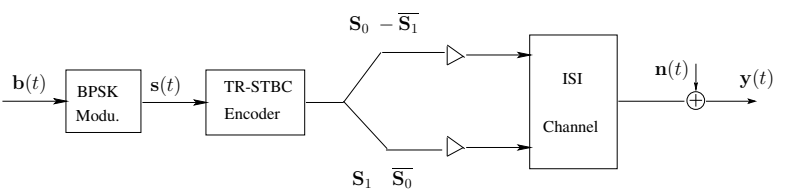

Figure 5. Diagram for the TR-STBC coded system.

\section{WLP for TR-STBC coded system}

An effective solution for meeting the high data rate and high quality of service requirements is to use space-time coding (STC) techniques $[11,12]$ employing multiple antennas. Space-time codes were originally designed for flat fading channels and did not consider the ISI introduced by frequency-selective channels. In [13], the two-antenna transmit diversity scheme [12] proposed by Alamouti was generalized for frequency selective channels, where the transmitted signals are coded on a block-to-block basis rather than a symbol-by-symbol basis. This is so-called time-reversal (TR) STBC.

In this section, we discuss how WLP can be applied to improve the performance of TR-STBC systems. Fig. 5 shows the baseband representation of the TR-STBC coded system under study. The information sequence $\mathbf{b}(t)$ is mapped into BPSK symbol sequence $\mathbf{s}(t)$. Unlike the Alamouti transmission scheme [12], which transmits two symbols from two antennas at a time, the TRSTBC encoder groups the symbols into two symbol blocks $\mathbf{S}_{0}$ and $\mathbf{S}_{1}$ at each antenna, each containing $N+1$ symbols. Two symbol blocks are simultaneously transmitted from two antennas. The data transmission is divided into two time frames. During the first time frame, $\mathbf{S}_{0}=$ $\left\{s_{0}(0), s_{0}(1), \ldots, s_{0}(N)\right\}$ is transmitted from the first antenna; and $\mathbf{S}_{1}=\left\{s_{1}(0), s_{1}(1), \ldots, s_{1}(N)\right\}$ is transmitted from the second antenna. During the second transmission frame, the first antenna transmits a time reversed, com- plex conjugated and sign inverted version of $\mathbf{S}_{1}$, denoted as $-\overline{\mathbf{S}_{1}}$; the second antenna transmits a time reversed, complex conjugated version of $\mathbf{S}_{0}$, denoted as $\overline{\mathbf{S}_{0}}$. The task of the receiver is to detect the transmitted information sequence $\mathbf{b}(t)$ given the received observation $\mathbf{y}(t)$ which is corrupted with ISI and AWGN noise $\mathbf{n}(t)$.

We consider the use of two transmit antennas and one receive antenna. The channel between the transmit antenna tx 0 and the receive antenna $\mathrm{rx} 0$ can be represented by a discrete-time filter as $h\left(z^{-1}\right)=\sum_{t=0}^{T-1} h_{t} z^{-t}$; the channel between the transmit antenna tx1 and the receive antenna rx0 can be represented by a discrete-time filter as $c\left(z^{-1}\right)=\sum_{t=0}^{T-1} c_{t} z^{-t}$. The channel coefficients are assumed to remain constant during the transmission of one block of data. The received signal during the first time frame $y_{0}(t)$ and the second time frame $y_{1}(t)$ can be formed as

$$
\underbrace{\left[\begin{array}{l}
y_{0}(t) \\
y_{1}(t)
\end{array}\right]}_{\mathbf{y}(t)}=\underbrace{\left[\begin{array}{cc}
h\left(z^{-1}\right) & c\left(z^{-1}\right) \\
c^{*}(z) & -h^{*}(z)
\end{array}\right]}_{\mathbf{H}\left(z, z^{-1}\right)} \underbrace{\left[\begin{array}{l}
s_{0}(t) \\
s_{1}(t)
\end{array}\right]}_{\mathbf{s}(t)}+\underbrace{\left[\begin{array}{l}
n_{0}(t) \\
n_{1}(t)
\end{array}\right]}_{\mathbf{n}(t)}
$$

The receiver algorithm is illustrated in Fig. 6. The signal vector $\mathbf{y}(t)$ is first filtered by the matched filter $\mathbf{H}^{*}\left(z, z^{-1}\right)$. The decoupled symbol streams $r_{0}(t)$ and $r_{1}(t)$ from the matched filter are each processed by an equalizer to derive an estimate of the transmitted symbol sequences $\tilde{s}_{0}(t)$ and $\tilde{s}_{1}(t)$, which are combined into a single symbol sequence, and demodulated to obtain an estimate of transmitted information bits $\left\{\hat{b}_{n}\right\}$. For a more detailed description of the TR-STBC algorithm and its application to the BFWA systems, readers are referred to [13] and [14].

The TR-STBC itself only decouples the symbol streams from two transmit antennas. It, however, does not resolve the ISI in each symbol stream. The ISI of course still needs to be handled by an equalizer, which is applied to the decoupled symbol streams $r_{0}(t), r_{1}(t)$ before making a decision on the transmitted symbols and bits. Fig. 7 compares the performance of the linear and widely linear MMSE equalizer, and the filter length is set to 5-tap in both cases. During each Monte-Carlo run, the block size $N+1$ is set to 250 for $\mathbf{S}_{0}$ and $\mathbf{S}_{1}$. The antenna correlation coefficient is set to 0.4 . The simulation curves are obtained by averaging the simulation results over 1000 channel realizations. Fig. 7 shows that the proposed WL equalizer performs better than the conventional linear equalizer in the TR-STBC coded BFWA system. The performance gain can be up to over $1 \mathrm{~dB}$.

\section{Widely Linear Approach to Turbo Equal- ization}

In this section, we apply WLP to systems with channel coding and derive a new approach to turbo equalization. The transmission system under study will be briefly described next. The information sequence $\left\{u_{n}\right\}$ is convo- 


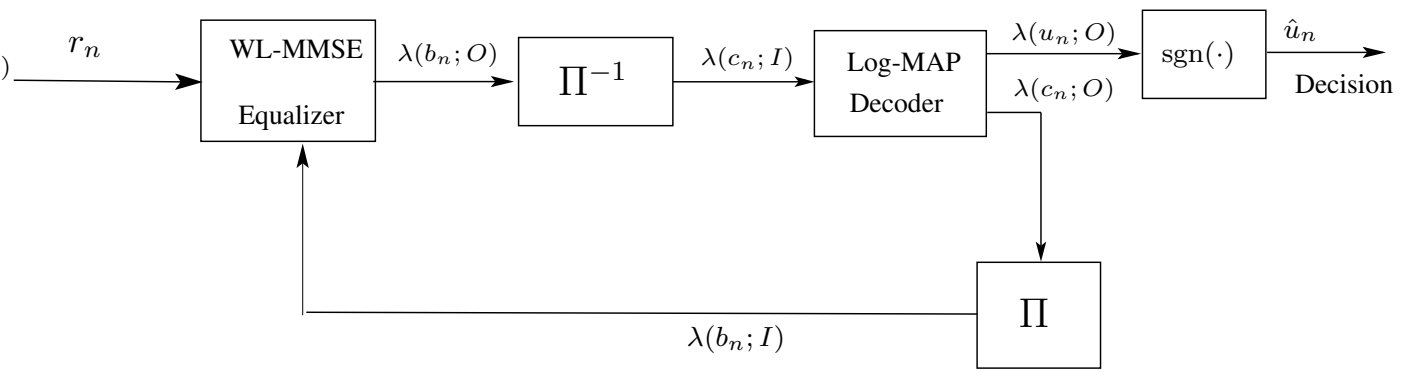

Figure 8. Proposed turbo equalization scheme.

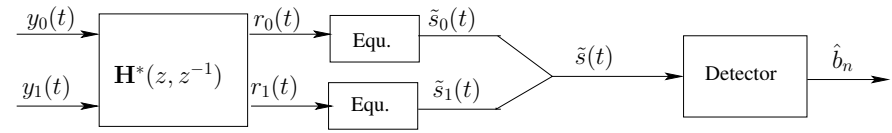

Figure 6. Receiver structure for the STBC coded BFWA system.

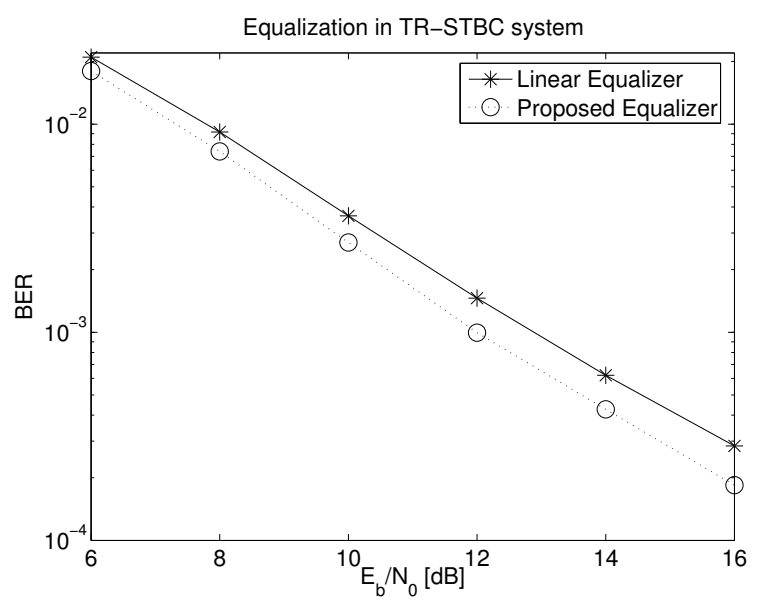

Figure 7. Performance of TR-STBC coded BFWA system. lutionally encoded into code bits $\left\{c_{n}\right\}$, which are subsequently interleaved and mapped into BPSK symbols $\left\{b_{n}\right\}$, which are transmitted over an ISI channel. A turbo equalizer is employed in the receiver as illustrated in Fig. 8. The received signal $r_{n}$ is passed to the equalizer, which computes the log-likelihood ratio (LLR) value of $b_{n}$, denoted by $\lambda\left(b_{n} ; O\right)$. The derived LLRs are deinterleaved to yield $\lambda\left(c_{n} ; I\right)$. Based on the soft input $\lambda\left(c_{n} ; I\right)$, a soft-input, soft-output (SISO) channel decoder computes the LLR of each information bit $\lambda\left(u_{n} ; O\right)$ and each coded bit $\lambda\left(c_{n} ; O\right)$. The former is used to make decision on the transmitted information bit $u_{n}$ at the final iteration, and the latter is interleaved to yield $\lambda\left(b_{n} ; I\right)$ which is the input to the equalizer at the next iteration. We use the notations $\lambda(\cdot ; I)$ and $\lambda(\cdot ; O)$ to denote the input and output ports of a SISO device, respectively. Several SISO algorithms can be used to compute the LLRs at the channel decoder output. For the purpose of this study, we consider the use of Log-MAP algorithm. The equalization algorithm will now be described in detail.

The interference canceled version of the received vector is given as $\mathbf{r}_{n}^{\prime}=\mathbf{r}_{n}-\mathbf{H} \overline{\mathbf{b}}_{n}=\mathbf{H}\left[\mathbf{b}_{n}-\overline{\mathbf{b}}_{n}\right]+\mathbf{v}_{n}$, where $\quad \mathbf{r}_{n}=\left[\begin{array}{llll}r_{n} & r_{n+1} & \ldots & r_{n+L-1}\end{array}\right]^{T}$, $\mathbf{v}_{n}=\left[\begin{array}{llll}v_{n} & v_{n+1} & \ldots & v_{n+L-1}\end{array}\right]^{T}$ denote the received vector and the noise vector, respectively; and $\mathbf{r}_{n}^{\prime}$ is the ISI canceled version of $\mathbf{r}_{n}$. The vector $\mathbf{b}_{n}$ and $\overline{\mathbf{b}}_{n}$ are defined respectively as $\mathbf{b}_{n}=$ $\left[\begin{array}{lllllll}b_{n-L+1} & \ldots & b_{n-1} & b_{n} & b_{n+1} & \ldots & b_{n+L-1}\end{array}\right]^{T}$, $\overline{\mathbf{b}}_{n}=\left[\begin{array}{lllllll}\bar{b}_{n-L+1} & \ldots & \bar{b}_{n-1} & 0 & \bar{b}_{n+1} & \ldots & \bar{b}_{n+L-1}\end{array}\right]^{T}$, where the latter contains the estimate of the interference symbols from the previous iteration. The derivation of $\overline{\mathbf{b}}_{n}$ will be given later on. The channel matrix is defined as

$\mathbf{H}=\left[\begin{array}{cccccccc}h_{L-1} & h_{L-2} & \ldots & h_{0} & 0 & 0 & \ldots & 0 \\ 0 & h_{L-1} & \ldots & h_{1} & h_{0} & 0 & \ldots & 0 \\ \vdots & \vdots & \vdots & & & & & \\ 0 & 0 & 0 & \ldots & h_{L-1} & h_{L-2} & \ldots & h_{0}\end{array}\right]$

In order to further suppress the residual interference in $\mathbf{r}_{n}^{\prime}$, an instantaneous linear MMSE filter is applied to $\mathbf{r}_{n}^{\prime}$, to obtain $z_{n}=\mathbf{w}_{n}^{H} \mathbf{r}_{n}^{\prime}$, where the filter coefficient vector $\mathbf{w}_{n}$ is chosen to minimize $e_{n}^{\mathrm{L}}=\mathrm{E}\left\{\left[b_{n}-\mathbf{w}_{n}^{H} \mathbf{r}_{n}^{\prime}\right]^{2}\right\}$. In 


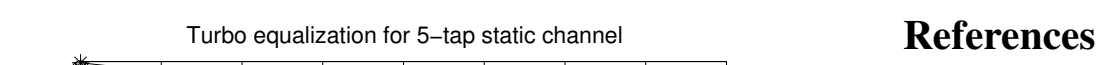

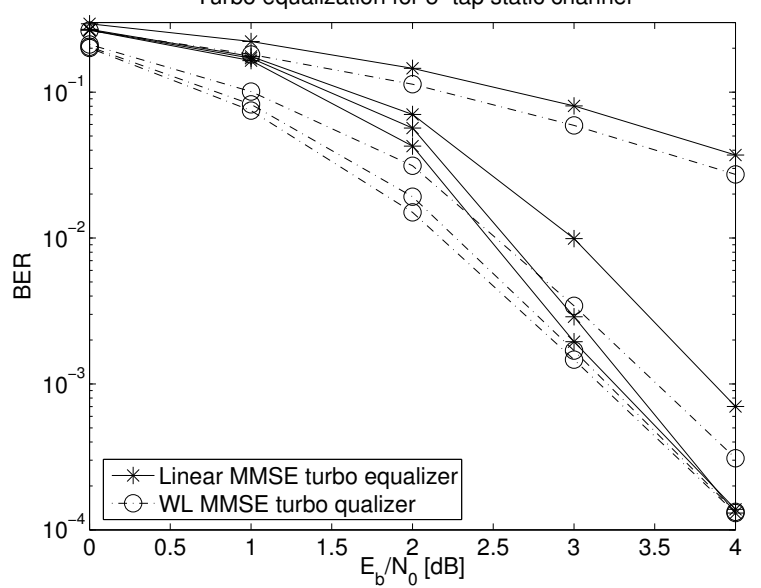

Figure 10. Comparison of different turbo equalization schemes for 5-tap static channel.

ISI channel. Fig. 9 shows the performance comparison for the BFWA SUI-3 channel. The curves are averaged over at least 500 channel realizations. In both cases, it takes only 3 stages for the algorithms to converge. When the system reaches convergence, a performance gain of $0.5 \mathrm{~dB}$ is observed by applying WLP comparing to the conventional linear MMSE approach at a BER between $10^{-3}$ and $10^{-4}$.

Finally, the two schemes are compared in Figure 10 for a static channel which has 5 taps and impulse response $h[n]=(2-0.4 j) \delta[n]+(1.5+1.8 j) \delta[n-1]+\delta[n-2]+$ $(1.2-1.3 j) \delta[n-3]+(0.8+1.6 j) \delta[n-4]$. The channel is normalized so that $P=\sum_{n=0}^{4}|h[n]|^{2}=1$. It takes 4 stages for both algorithms to converge. Compared to the initial stage with one time equalization and Log-MAP decoding, the subsequent turbo equalization stages achieve much better performance. The WL turbo equalizer generally outperforms its linear counterpart except at $E_{b} / N_{0}=4$ $\mathrm{dB}$ upon convergence. The gain is most obvious at the second stage.

\section{Conclusions}

In this paper, we apply widely linear processing technique to frequency selective channels and propose several WL equalization schemes, which are designed under a revised mean square error criterion. The WL schemes are evaluated in the single-input, single-output, as well as the TRSTBC coded BFWA systems and demonstrate superior performance compared to the conventional equalizers. A WLP approach to turbo equalization is also introduced and is shown to achieve better performance than conventional filter based turbo equalization. The proposed WLP based schemes can be employed in practical systems in order to combat ISI more effectively and improve the system performance and capacity.
[1] I. Koffman. "Broadband fixed wireless access solutions based on OFDM access in IEEE802.16". IEEE Coтmunication Magazine, vol. 40, pp. 96-103, April, 2002.

[2] P. Schreier, L. Scharf, C. Mullis. "Detection and estimation of improper complex random signals", IEEE Transactions on Information Theory, vol. 51, no. 1, pp. 306-312, January 2005.

[3] F. Neeser, J. Massey. "Proper complex random processes with applications to information theory", IEEE Transactions on Information Theory, vol. 39, no. 4, pp. 1293-1302, July 1993.

[4] B. Picinbono, P. Chevalier. "Widely linear estimation with complex data", IEEE Transactions on Signal Processing, vol. 43, no. 8, pp. 2030-2033, August 1995.

[5] S. Buzzi, M. Lops, A. Tulino. "A new family of MMSE multiuser receivers for interference suppression in DS/CDMA systems employing BPSK modulation". IEEE Transactions on Communications, vol. 49, no. 1, pp. 154-167, Jan. 2001.

[6] W. Gerstacker, R. Schober, A. Lampe. "Receivers with widely linear processing for frequency-selective channels". IEEE Transactions on Communications, vol. 51, no. 9, pp. 1512-1523, Sept. 2003.

[7] J. Proakis. Digital Communications, 3rd edition, McGrawHill, 1995.

[8] S. Haykin. Adaptive Filter Theory, 4th edition, Prentice Hall, 2002.

[9] IEEE 802.16 Working Group on Broadband Wireless Access Standards. available at http://grouperieee.org/groups/802/16/. 2002.

[10] V. Erceg. "An empirically based path loss model for wireless channels in suburban environments". IEEE JSAC, vol. 17, no. 7, pp. 1205-1211, July 1999.

[11] V. Tarokh, N. Seshadri, A. Calderbank. "Space-time codes for high data rates wireless communications: performance criterion and code construction". IEEE Transactions on Information Theory, vol. 44, pp. 744-765, March 1998.

[12] A. Alamouti. "A simple transmit diversity technqiue for wireless communications". IEEE Journal on Selected Areas in Communications, vol. 16, no. 8, pp. 1451-1458, Oct. 1998.

[13] E. Lindskog, A. Paulraj. "A transmit diversity scheme for channels with intersymbol interference". IEEE International Conference on Communications, pp. 307-311, June 2002.

[14] P. Xiao, R. Carrasco, I. Wassell. "Time reversal space-time block coding for FWA systems". International Conference on Wireless and Mobile Communications, July 2006.

[15] M. Tuchler, R. Koetter, A. Singer. "Turbo equalization: principles and new results", IEEE Transactions on Communications, vol. 50, pp. 754-767, May 2002. 